\title{
ADAPTAÇÃO E VALIDAÇÃO DA ESCALA TOULOUSIANA DE COPING A ADOLESCENTES
}

\author{
| Manuela Amaral-Bastos ${ }^{1}$; Beatriz Araújo²; Alexandre Castro Caldas ${ }^{3} \mid$
}

\section{RESUMO}

CONTEXTO: As estratégias de coping face a acontecimentos adversos são multidimensionais e articulam os campos comportamental, cognitivo e afetivo. O conhecimento das estratégias utilizadas pelos adolescentes proporciona informação para uma intervenção mais eficaz. OBJETIVO: Adaptar e avaliar as propriedades psicométricas da Escala Toulousiana de Coping numa amostra de 291 adolescentes portugueses que frequentam o $3^{\circ}$ Ciclo dos Ensinos Básico e Secundário, em dois Agrupamentos de Escolas; e analisar a influência do género e idade no Coping.

METODOLOGIA: Realizamos um estudo transversal, quantitativo. Para aferir as propriedades psicométricas do instrumento verificamos a confiabilidade e validade dos resultados, através duma análise fatorial de componentes principais com rotação Varimax dos itens da escala e do cálculo do coeficiente Alpha de Cronbach. Recorremos à análise estatística inferencial para estudar a relação entre variáveis.

RESULTADOS: A Escala Toulousiana de Coping apresenta caraterísticas psicométricas adequadas para este grupo de adolescentes ( $\alpha$ Cronbach .85). O estudo da dimensionalidade identificou cinco subescalas: Controlo; Suporte Social; Retraimento, Conversão e Aditividade; Distração Social e Recusa. O Coping Global é, em média, ligeiramente mais elevado nos rapazes. No entanto, as estratégias de controlo, suporte e distração social são utilizadas de forma idêntica por rapazes e raparigas. Por sua vez, as estratégias de retraimento, conversão e aditividade são tendencialmente mais utilizadas pelas raparigas e a recusa pelos rapazes.

CONCLUSÕES: A Escala Toulousiana de Coping é um instrumento fiável e útil para os enfermeiros, com vista à implementação de cuidados preferencialmente proativos, personalizados e direcionados para os problemas detetados em adolescentes.

PALAVRAS-CHAVE: Adolescente; Estudos de validação; Adaptação psicológica

\section{RESUMEN}

\section{“Adaptación y Validación de la Escala Toulousiana de Coping a adolescentes"}

CONTEXTO: Las estrategias de coping frente a eventos adversos son multidimensionales y articulan los campos conductual, cognitivo y afectivo. El conocimiento de las estrategias utilizadas por adolescentes proporciona información para una intervención más eficaz.

OBJETIVO: Adaptar y evaluar las propiedades psicométricas de la Escala Toulousiana de Coping en una muestra de 291 adolescentes portugueses que asisten el $3^{\circ}$ ciclo de Educación Básica y Secundaria, en dos grupos de escuelas; analizar la influencia del género y edad en el Coping.

METODOLOGIA: Llevamos a cabo un estudio transversal, cuantitativo. Para aferir las propiedades psicométricas del instrumento verificamos la fiabilidad y validez de los resultados, por medio de análisis factorial de componentes principales con rotación varimax de los ítens del cuestionario y el cálculo del Alpha de Cronbach. Recurrimos al análisis estadístico inferencial para estudiar la relación entre variables.

RESULTADOS: La Escala Toulousiana de Coping presenta características psicométricas adecuadas para estos adolescentes (a Conbach .85). El estudio de la dimensionalidad ha identificado cinco subescalas: control, soporte social, retraimiento, conversión y adictividad; distracción social y recusa. El Coping Global es, en media, ligeramente más alto en los chicos. Las estrategias de control, soporte y distracción social son utilizadas de forma idéntica por chicos y chicas. Las estrategias de retraimiento, conversión y aditividad tienden a ser más utilizadas por las chicas y la recusa por los chicos.

CONCLUSIONES: La Escala Toulousiana de Coping es un instrumento fiable y útil para los enfermeros, visando la implementación de cuidados preferentemente proactivos, personalizados y direccionados a los problemas detectados en adolescentes.

DESCRIPTORES: Adolescente; Estudios de validación; Adaptación psicológica

\section{ABSTRACT \\ "Adaptation and Validation of Toulousiana Coping Scale for adolescents"}

BACKGROUND: The coping strategies faces to adverse events are multidimensional who articulate the behavioral, cognitive and affective fields. Knowledge of strategies used by adolescents provides information for more effective intervention.

AIMS: To adapt and evaluate the psychometric properties of the Toulousiana Coping Scale in a sample of 291 Portuguese adolescents who attend the 3rd cycle of Basic and Secondary Education, in two school groups; and analyze the influence of gender and age on strategies of coping.

METHODS: We conducted a transversal study with a quantitative approach. To assess the psychometric properties of the instrument assessing the reliability and validity of results, through a factorial analysis of the principal components with Varimax rotation of the questionnaire items and the calculation of Cronbach's Alpha. We appealed also the inferential statistical analysis to study the relationship between variables.

RESULTS: The Toulousiana Coping Scale has adequate psychometrics characteristics for this group of adolescents (Cronbach a .85). The study of dimensionality identified five subscales: Control, Social Support; Withdrawal, conversion and additivity; Social Distraction and Refusal. The Global Coping is on average slightly higher in boys. However, control strategies, support and social distraction are used in the same way for boys and girls. In turn, the withdrawal strategies, conversion and additivity tend to be most used by girls and the refusal by the boys.

CONCLUSIONS: The Toulousiana Coping Scale it is a reliable measurement instrument and useful for nurses in order to implement care preferably proactive, personalized and directed to the problems detected in adolescents.

\section{KEYWORDS: Adolescent; Validation studies; Adaptation, psychological}

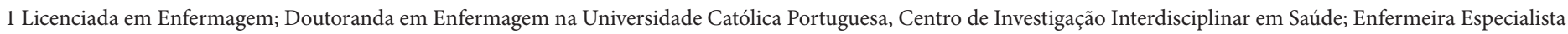

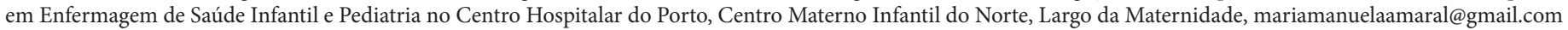

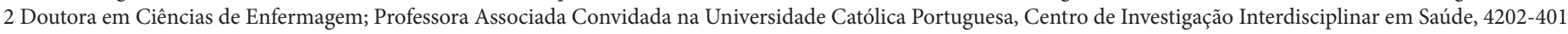
Porto, Portugal, baraujo@porto.ucp.pt

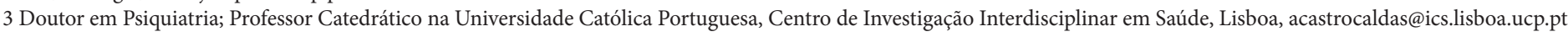

Submetido em 20-03-2015 - Aceite em 30-11-2015

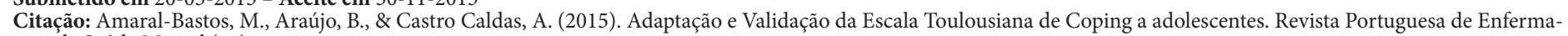
gem de Saúde Mental (14), 55-63.
} 


\section{INTRODUÇÃO}

O termo coping é de origem anglo-saxónica, não traduzível para português mas pode significar modo de lidar ou de enfrentar uma determinada adversidade. Neste estudo optamos por utilizar a palavra original.

Os estudos sobre coping tiveram a sua origem nas pesquisas sobre stress. Ao longo do tempo várias gerações de investigadores têm-se debruçado sobre o coping, olhando-o de diversas perspetivas (modelos de coping, estilos, estratégias, resultados, entre outras) e enriquecendo o conceito com os aportes que a investigação tem produzindo (Antoniazzi, Dell'Aglio e Bandeira, 1998; Diniz e Zanini, 2010; Faro e Pereira, 2013). O coping é considerado "um conjunto de esforços, cognitivos e comportamentais, utilizado pelos indivíduos com o objetivo de lidar com demandas específicas, internas ou externas, que surgem em situações de stress e são avaliadas como sobrecarregando ou excedendo os seus recursos pessoais" (Lazarus \& Folkman, 1984, p. 141). Os autores completam o conceito como sendo um processo cognitivo que permite ao indivíduo dar um sentido à situação em função dos recursos que ele pode mobilizar, para obter um ajustamento adequado.

Segundo a teoria de coping de Lazarus (1992), independentemente do indivíduo procurar estratégias que reduzam o nível de emoções negativas como a ansiedade e angústia ou a gestão e alteração da fonte de stress, a maior parte das pessoas recorre a cerca de oito estratégias de coping que passamos a descrever: (i) coping confrontativo - recurso a esforços agressivos de alteração da situação com um certo grau de hostilidade e risco; (ii) distanciamento - os esforços utilizados são de nível cognitivo de desprendimento e minimização da situação, o indivíduo apercebe-se da situação geradora do stress mas não quer lidar com o seu significado emocional; (iii) auto-controlo - os esforços de regulação dos próprios sentimentos e ações; (iv) suporte social - o individuo procura suporte informativo, tangível e emocional; ( $v$ ) aceitar a responsabilidade - a pessoa reconhece o problema e concomitantemente investe na tentativa de reorganizar a situação; (vi) fuga-evitamento - os esforços cognitivos e comportamentais são usados para escapar ou evitar o problema, o que pode gerar repercussões negativas a longo prazo na saúde do indivíduo, conduzindo a um bem-estar emocional apenas a curto prazo; (vii) resolução planeada do problema - os esforços são focados sobre o problema com o objetivo de alterar a situação; e (viii) reavaliação positiva - desenvolvem-se esforços de criação de significados positivos, focando o crescimento pessoal, tendo, muitas vezes, uma dimensão religiosa.
A eficácia das estratégias de coping varia ao longo da vida do individuo, difere dos processos automáticos adaptativos e os próprios critérios de eficácia devem ser avaliados de forma multidimensional, pois uma estratégia de coping só é adequada se permitir dominar ou diminuir o impacto da ameaça. O coping é a variável intermediária entre um acontecimento e os resultados ou consequências desses acontecimentos. (Pais-Ribeiro, 2004)

No decorrer da investigação, vários instrumentos de medida foram construídos com o objetivo de avaliar o coping. Partindo da análise das escalas produzidas para avaliação do coping, Esparbès, Sordes-Ader e Tap (1993) propuseram uma nova escala multidimensional que articula os campos comportamental (ação), cognitivo (informação) e afetivo (emoção). Da interação destes três campos emergem seis estratégias: focalização, suporte social, retraimento, conversão, controlo e recusa. Estas, por sua vez, deram origem a 18 dimensões que apresentamos no Quadro 1. Esta escala foi posteriormente adaptada culturalmente e avaliadas as propriedades psicométricas para a população portuguesa com a designação de Escala Toulousiana de Coping. (Tap, Costa e Alves, 2005)

Quadro 1 - Relação entre Campos e Estratégias de Coping

\begin{tabular}{|c|c|c|c|}
\hline \multicolumn{4}{|c|}{ Campos } \\
\hline Estratégias & $\begin{array}{l}\text { Comportamental } \\
\text { (Ação) }\end{array}$ & $\begin{array}{c}\text { Cognitivo } \\
\text { (Informação) }\end{array}$ & $\begin{array}{c}\text { Afetivo } \\
\text { (Emoção) }\end{array}$ \\
\hline Focalização & Focalização Ativa & Focalização Cognitiva & $\begin{array}{c}\text { Focalização } \\
\text { Emocional }\end{array}$ \\
\hline Suporte Social & Cooperação & $\begin{array}{l}\text { Suporte Social Infor- } \\
\text { mativo }\end{array}$ & $\begin{array}{c}\text { Suporte Social } \\
\text { Emocional }\end{array}$ \\
\hline Retraimento & $\begin{array}{l}\text { Retraimento Social } \\
\text { e Comportamental }\end{array}$ & Retraimento Mental & Aditividade \\
\hline Conversão & $\begin{array}{l}\text { Conversão Com- } \\
\text { portamental }\end{array}$ & Aceitação & $\begin{array}{c}\text { Conversão } \\
\text { pelos Valores }\end{array}$ \\
\hline Controlo & $\begin{array}{l}\text { Regulação Ativi- } \\
\text { dades }\end{array}$ & $\begin{array}{l}\text { Controlo Cognitivo e } \\
\text { Planificação }\end{array}$ & $\begin{array}{l}\text { Controlo Emo- } \\
\text { cional }\end{array}$ \\
\hline Recusa & Distração & Denegação & Alexitimia \\
\hline
\end{tabular}

Retirado de Tap, et al., 2005

Na estratégia:

(i) "Focalização" - a pessoa foca-se no problema e na forma de o resolver (focalização ativa), analisa a situação (focalização cognitiva) ou é invadido pelas emoções (focalização emocional);

(ii) "Suporte Social" - a pessoa sente necessidade de ajuda e pode procurá-la através de um trabalho conjunto (cooperação), buscando esclarecimentos ou conselhos (suporte social informativo), ou quem a ouça, conforte e apoie (suporte social emocional);

(iii) "Retraimento" - significa uma quebra nas relações sociais e que pode traduzir-se por evitar contactos, 
levando a pessoa a isolar-se (retraimento social e comportamental), podem existir esforços para esquecer, negar-se a pensar no problema refugiando-se num mundo imaginário (retraimento mental) e pode também verificar-se a presença de comportamentos compensatórios como a ingestão de fármacos, comida ou drogas (aditividade);

(iv) "Conversão" implica mudança, podendo ser uma mudança de comportamento face ao problema (conversão comportamental), uma aceitação da situação (aceitação) ou confiar em Deus, adotando uma filosofia de vida ou ideologia religiosa (conversão pelos valores); (v) "Controlo" significa que a pessoa toma as rédeas da situação refletindo nas decisões a tomar, evitando precipitar-se (regulação das atividades), planifica as suas ações (controlo cognitivo e planificação) ou controla as suas emoções de forma a que os outros não se apercebam (controle emocional);

(vi) "Recusa" significa que a pessoa pode fazer de conta que o problema não existe, distraindo-se ou fazendo qualquer coisa mais agradável (distração), pode "esquecer" o problema (denegação) ou pode ter dificuldade em expressar as suas emoções e sentimentos (alexitimia) (Tap, et al., 2005)

A Escala Toulousiana de Coping (ETC) tem sido objeto de vários estudos (Alves, 2003; Nunes, 2001; Tap, et al., 2005). Quando a selecionamos já se encontrava traduzida para português e validada numa população de 1000 jovens adultos, com idades compreendidas entre os $18 \mathrm{e}$ os 28 anos (Tap, Costa e Alves, 2005). A análise fatorial efetuada permitiu encontrar 5 subescalas: Controlo; Retraimento, Conversão e Aditividade (RCA); Suporte Social, Distração Social; e Recusa. A fidelidade, avaliada através do Alpha de Cronbach, foi de 0,78 (Tap, Costa e Alves, 2005). Ao longo do texto, passamos a denominar este estudo como estudo original.

Este estudo tem como objetivo adaptar e avaliar as propriedades psicométricas da Escala Toulousiana de Coping em adolescentes dos 12 aos 18 anos e analisar a influência das variáveis género e idade no Coping desses adolescentes. Tem, ainda, em vista disponibilizar um instrumento multidimensional, a ser utilizado por enfermeiros e por outros profissionais das áreas da saúde e da educação, que permita identificar estratégias de coping que possam contribuir para a prevenção de comportamentos de risco em situações adversas. Neste estudo seguimos as orientações teórico-metodológicas de Almeida e Freire (2008) e de Pais-Ribeiro (2010) relativamente aos procedimentos de validação de escalas.

\section{METODOLOGIA}

\section{Participantes}

Recorremos a uma amostra não probabilística de conveniência constituída por 291 adolescentes a frequentar o $3^{\circ}$ Ciclo do Ensino Básico e o Ensino Secundário em dois Agrupamentos de Escolas da zona norte de Portugal.

\section{Instrumento}

A ETC é um questionário de autorrelato constituído por 51 itens, distribuídos por cinco subescalas, designadamente: Controlo; Retraimento, Conversão e Aditividade; Distracção Social; Suporte Social e Recusa. Cada item é respondido numa escala tipo Likert, com 5 opções de resposta: (1) Nunca; (2) Quase nunca; (3) Indeciso; (4) Quase sempre; (5) Sempre (Tap, Costa e Alves, 2005).

\section{Procedimentos}

Realizamos um estudo transversal, quantitativo com recolha de dados através de questionário de autopreenchimento numa amostra de conveniência. A colheita de dados decorreu no período compreendido entre dezembro de 2013 e abril de 2014 e foi efetuada em dois Agrupamentos de Escolas da zona norte de Portugal, após a obtenção das autorizações das respetivas Direções e dos pais/encarregados de educação dos participantes no estudo.

Foi também solicitada autorização ao Gabinete de Estatística e Planeamento da Educação para Monitorização de Inquéritos em Meio Escolar do Ministério da Educação. Relativamente aos adolescentes, recorremos ao consentimento presumido.

Mesmo com autorização prévia dos pais/encarregados de educação, foi sempre assegurada a participação voluntária dos adolescentes.

Numa fase inicial efetuamos um pré-teste com 7 alunos de 14 anos a fim de aferir a compreensão do conteúdo dos itens.

A análise foi efetuada em sala. Após identificação dos itens suscetíveis de serem melhorados, foram discutidos um a um, tendo sido propostas alterações em 7 itens que apresentamos no Quadro 2. 
Quadro 2 - Alterações Propostas a Alguns Itens da ETC

\begin{tabular}{|c|c|c|c|}
\hline \multirow{2}{*}{$\begin{array}{c}\text { Item } \\
\mathrm{n}^{\circ}\end{array}$} & \multicolumn{2}{|c|}{ Redação } & \multirow{2}{*}{$\begin{array}{c}\text { Item } \\
\mathrm{n}^{\circ}\end{array}$} \\
\hline & Texto original & Texto após reformulação & \\
\hline 4 & $\begin{array}{l}\text { Trabalho em cooperação } \\
\text { com outras pessoas para me } \\
\text { esquecer }\end{array}$ & $\begin{array}{l}\text { Trabalho em conjunto com } \\
\text { outras pessoas para me } \\
\text { esquecer }\end{array}$ & 3 \\
\hline 12 & $\begin{array}{l}\text { É-me difícil utilizar palavras } \\
\text { para descrever o que ex- } \\
\text { perimento face a uma situação } \\
\text { difícil }\end{array}$ & $\begin{array}{l}\text { Face a uma situação difícil, } \\
\text { tenho dificuldade em encontrar } \\
\text { palavras para dizer o que sinto }\end{array}$ & 11 \\
\hline 15 & $\begin{array}{l}\text { Sinto agressividade face a } \\
\text { outrem }\end{array}$ & $\begin{array}{l}\text { Sinto-me agressivo para com } \\
\text { outras pessoas }\end{array}$ & 14 \\
\hline 24 & $\begin{array}{l}\text { Procuro encontrar uma } \\
\text { filosofia de vida para fazer face } \\
\text { à situação }\end{array}$ & $\begin{array}{l}\text { Procuro encontrar uma filoso- } \\
\text { fia de vida (sentido, valor) para } \\
\text { fazer face à situação }\end{array}$ & 23 \\
\hline 34 & $\begin{array}{l}\text { Vou ao cinema ou vejo tele- } \\
\text { visão para pensar menos nisso }\end{array}$ & $\begin{array}{l}\text { Vou ao cinema, internet, vejo } \\
\text { televisão ou faço jogos para } \\
\text { pensar menos nisso }\end{array}$ & 33 \\
\hline 35 & $\begin{array}{l}\text { Penso nas experiências suscet- } \\
\text { íveis de me ajudarem a fazer } \\
\text { face à situação }\end{array}$ & $\begin{array}{l}\text { Penso nas experiências capazes } \\
\text { de me ajudarem a fazer face à } \\
\text { situação }\end{array}$ & 34 \\
\hline 38 & $\begin{array}{l}\text { Pergunto às pessoas que } \\
\text { tiveram uma situação similar, } \\
\text { que tipo de resposta deram }\end{array}$ & $\begin{array}{l}\text { Pergunto às pessoas que res- } \\
\text { posta deram quando passaram } \\
\text { por uma situação semelhante }\end{array}$ & 37 \\
\hline
\end{tabular}

$\mathrm{Na}$ análise dos dados recorremos ao Statistical Package for Social Sciences (SPSS), versão 22 para Windows. Para efetuarmos a avaliação psicométrica da ETC em adolescentes, adotamos os mesmos procedimentos dos autores da escala. Assim, procedemos à análise factorial exploratória dos itens em componentes principais, com rotação ortogonal pelo método varimax, para verificar a validade de construto; determinação do coeficiente Alpha de Cronbach para avaliar a consistência interna da escala total e das subescalas; e cálculo do teste t de student para analisar a influência das variáveis género e idade no Coping Global.

Realizamos, também, o Teste de Kaiser-Meyer-Olkin (KMO) e o Teste de Esfericidade de Bartlett (TEB) para aferir a adequação da amostra para a efetivação da análise fatorial. Para que esta seja harmoniosa e fiável tínhamos como pressuposto não aceitar qualquer saturação abaixo de 30\% (Almeida e Freire, 2008).

\section{RESULTADOS}

A amostra de 291 adolescentes frequenta o $3^{\circ}$ Ciclo do Ensino Básico (7 Ano, 26.5\%; 8 Ano, 37.8\%; 9 Ano, $32.3 \%$ ) e o Ensino Secundário (10 Ano, 0.7\%; e $11^{\circ}$ Ano, 2.7\%), tem idades compreendidas entre os 12 e os 18 anos (12-14 anos, $82.8 \%$ e $>15$ anos, $17.2 \%$ ), e em média 13.7 anos e um desvio padrão de 1.13 anos. É constituída maioritariamente por adolescentes do sexo feminino (67.4\%) e a viver com a família (97.6\%). O agregado familiar é na sua maioria constituído pelos pais e irmãos (80.1\%).
Dos inquiridos, $23.7 \%$ refere reprovações ao longo do seu percurso escolar. O Teste KMO e o Teste de Esfericidade de Bartlett $(\mathrm{TEB}<.05)$ permitiram aferir a adequação da amostra para a realização da análise fatorial $(\mathrm{KMO}=.78 ; \mathrm{TEB}=4566.900, \mathrm{p}<.001)$.

O estudo da dimensionalidade da ETC foi efetuado através duma análise fatorial de componentes principais com rotação ortogonal pelo método varimax e normalização de Kaiser dos 51 itens da escala. Dessa análise, retiveram-se os itens com valor-próprio igual ou superior à unidade e as saturações fatoriais dos itens iguais ou superiores a .30, à exceção do item 5 que apresenta uma carga fatorial de .29 .

Os autores decidiram não eliminar o item "Peço a Deus para me ajudar" pelo seu significado concetual e contributo para o fator. Da análise identificaram-se cinco subescalas (ou fatores), a saber: Controlo (14 itens), Suporte Social (11 itens); RCA (14 itens); Distração Social (6 itens) e Recusa (6 itens). Estes cinco fatores explicam no seu conjunto $37.1 \%$ da variância total da escala. Ao fator 1 está associada $13.9 \%$ da variância total, o valor próprio de 7.1 e é saturado sobretudo pelos 14 itens relacionados com o "Controlo". Ao fator 2 está associada $8.6 \%$ da variância total, o valor próprio de 4.4 e é saturado sobretudo pelos 11 itens relacionados com o Suporte Social. Ao fator 3 está associada $5.3 \%$ da variância total, o valor próprio é de 2.7 e é saturado sobretudo pelos 14 itens relacionados com o "RCA". Por sua vez, ao fator 4 está associada $4.9 \%$ da variância total, o valor próprio de 2.5 e é saturado sobretudo pelos 6 itens relacionados com a "Recusa". Por fim, ao fator 5 está associada $4.4 \%$ da variância total, o valor próprio de 2.3 e é saturado sobretudo pelos 6 itens relacionados com "Distração Social".

$\mathrm{Na}$ Tabela 1 apresentamos os valores resultantes da análise da dimensionalidade da ETC, os valores-próprios e a percentagem de variância explicada por cada fator, as comunalidades (h2), o total de variância explicada, a medida de adequação da amostra através dos Testes de Kaiser-Meyer-Olkin e de Esfericidade de Bartlett. 
Tabela 1 - Resultados da Análise Fatorial da ETC

\begin{tabular}{|c|c|c|c|c|c|c|}
\hline \multirow{2}{*}{ Itens } & \multicolumn{5}{|c|}{ Fatores } & \multirow{2}{*}{ h2 } \\
\hline & 1 & 2 & 3 & 4 & 5 & \\
\hline 1 - Enfrento a situação. & .47 & & & & & .35 \\
\hline 7 - Aceito o problema se ele for inevitável. & .59 & & & & & .36 \\
\hline 8 - Tento não entrar em pânico. & .57 & & & & & .34 \\
\hline 15 - Faço qualquer coisa mais agradável. & .40 & & & & & .43 \\
\hline 16 - Analiso a situação para melhor a compreender. & .61 & & & & & .45 \\
\hline 18 - Ataco o problema de frente. & .49 & & & & & .39 \\
\hline 25 - Aprendo a viver com o problema. & .39 & & & & & .21 \\
\hline 26 - Controlo as minhas emoções. & .52 & & & & & .41 \\
\hline 28 - Sei o que tenho que fazer e redobro os meus esforços para o alcançar. & .52 & & & & & .46 \\
\hline 30 - Resisto ao desejo de agir até que a situação me permita. & .31 & & & & & .22 \\
\hline 36 - Vou diretamente ao problema. & .47 & & & & & .37 \\
\hline 39 - Ajudo os outros como gostaria de ser ajudado. & .47 & & & & & .42 \\
\hline 43 - Aceito a ideia de que é necessário que eu resolva o problema. & .67 & & & & & .50 \\
\hline 45 - Defino objetivos a alcançar. & .56 & & & & & .38 \\
\hline 2 - Peço conselhos a profissionais (professores, médicos, psicólogos, etc) & & .63 & & & & .43 \\
\hline 5 - Peço a Deus para me ajudar. & & .29 & & & & .14 \\
\hline 10 - Faço um plano de ação e tento aplica-lo. & & .53 & & & & .41 \\
\hline 17 - Sinto necessidade de partilhar com os que me são próximos o que sinto. & & .65 & & & & .45 \\
\hline 19 - Discuto o problema com os meus pais. & & .51 & & & & .38 \\
\hline 21 - Procuro atividades coletivas. & & .51 & & & & .36 \\
\hline 34 - Penso nas experiências capazes de me ajudarem a fazer face à situação. & & .45 & & & & .37 \\
\hline 35 - Procuro a ajuda dos meus amigos para acalmar a minha ansiedade. & & .57 & & & & .36 \\
\hline 37 - Pergunto às pessoas que resposta deram quando passaram por uma situação semelhante. & & .58 & & & & .37 \\
\hline 50 - Reflito nas estratégias que poderei utilizar para melhor resolver o problema. & & .43 & & & & .33 \\
\hline 51 - Procuro a simpatia e o encorajamento dos outros. & & .52 & & & & .41 \\
\hline 6 - Evito encontrar-me com pessoas. & & & .39 & & & .20 \\
\hline 9 - Mudo a minha forma de viver. & & & .43 & & & .43 \\
\hline 11 - Face a uma situação difícil tenho dificuldade em encontrar palavras para dizer o que sinto. & & & .51 & & & .39 \\
\hline 14 - Sinto-me agressivo para com outras pessoas. & & & .38 & & & .20 \\
\hline 20 - Esqueço os meus problemas tomando medicamentos. & & & .41 & & & .37 \\
\hline 22 - Refugio-me no imaginário ou no sonho. & & & .61 & & & .42 \\
\hline 23 - Procuro encontrar uma filosofia de vida (sentido, valor) para fazer face à situação. & & & .42 & & & .34 \\
\hline 24 - Acontece-me não fazer o que tinha decidido. & & & .51 & & & .33 \\
\hline 27 - Mudo o meu comportamento. & & & .46 & & & .29 \\
\hline 32 - Sinto-me culpado. & & & .57 & & & .36 \\
\hline 38 - Tomo drogas ou fumo para acalmar a minha angústia. & & & .32 & & & .21 \\
\hline 42 - Afasto-me dos outros. & & & .58 & & & .40 \\
\hline 44 - Modifico as minhas ações em função do problema. & & & .37 & & & .31 \\
\hline 48 - Sinto-me invadido pelas minhas emoções. & & & .59 & & & .42 \\
\hline 3 - Trabalho em conjunto com outras pessoas para me esquecer. & & & & .35 & & .27 \\
\hline 4 - Tento não pensar no problema. & & & & .55 & & .38 \\
\hline 13 - Recuso-me a admitir o problema. & & & & .41 & & .48 \\
\hline 33 - Vou ao cinema, internet, vejo televisão ou faço jogos para pensar menos nisso. & & & & .64 & & .46 \\
\hline 40 - Procuro a todo o custo pensar noutra coisa. & & & & .76 & & .60 \\
\hline 49 - Centro-me noutras atividades para me distrair. & & & & .62 & & .45 \\
\hline 12 - Não penso nos problemas quando eles se colocam. & & & & & .48 & .34 \\
\hline 29 - Chego a não sentir nada quando chegam as dificuldades. & & & & & .55 & .40 \\
\hline 31 - Reajo como se o problema não existisse. & & & & & .52 & .44 \\
\hline 41 - Brinco com a situação em que me encontro. & & & & & .52 & .29 \\
\hline 46 - As minhas emoções desaparecem tão rapidamente como aparecem. & & & & & .62 & .42 \\
\hline 47 - Digo a mim próprio que este problema não tem importância. & & & & & .61 & .47 \\
\hline Total de variância explicada $-37.1 \%$ & 13.9 & 8.6 & 5.3 & 4.9 & 4.4 & \\
\hline Valor próprio & 7.1 & 4.4 & 2.7 & 2.5 & 2.3 & \\
\hline
\end{tabular}


Para avaliar a fidelidade através da consistência interna do instrumento calculamos o coeficiente Alpha de Cronbach quer para cada item, quer para a globalidade dos itens. Da análise dos resultados verificamos que os valores da correlação de cada item com o total da escala, oscilam entre .29 e .54 . Todos os itens cumprem o critério: correlações superiores a .20 (Almeida \& Freire, 2008). Tomando os valores por itens, verificamos que a média varia entre 1.4 e 4.2 e o desvio-padrão em torno da unidade (.74 e 1.44). Constatamos, ainda, que os coeficientes Alpha de Cronbach relativos a cada item, com o total da escala (Alpha global $=.85$ ), oscilam entre $.84 \mathrm{e}$ .85 , localizando-se na categoria de consistência interna "Boa" (Pestana e Gageiro, 2008).

Estes valores indicam uma boa correlação entre todos os itens e uma boa homogeneidade dos itens e revelam que os itens em conjunto contribuem para as estratégias de coping. Efetuamos também a análise da consistência interna da ETC e das subescalas cujos resultados apresentamos na Tabela 2. A par da média e do desvio-padrão, descrevemos a respetiva correlação do item com o total da sua escala de pertença (coeficiente corrigido) e também o valor do Alpha da subescala se esse mesmo item fosse eliminado, considerando a amostra total.

Tabela 2 - Análise da Consistência Interna da ETC e das Subescalas

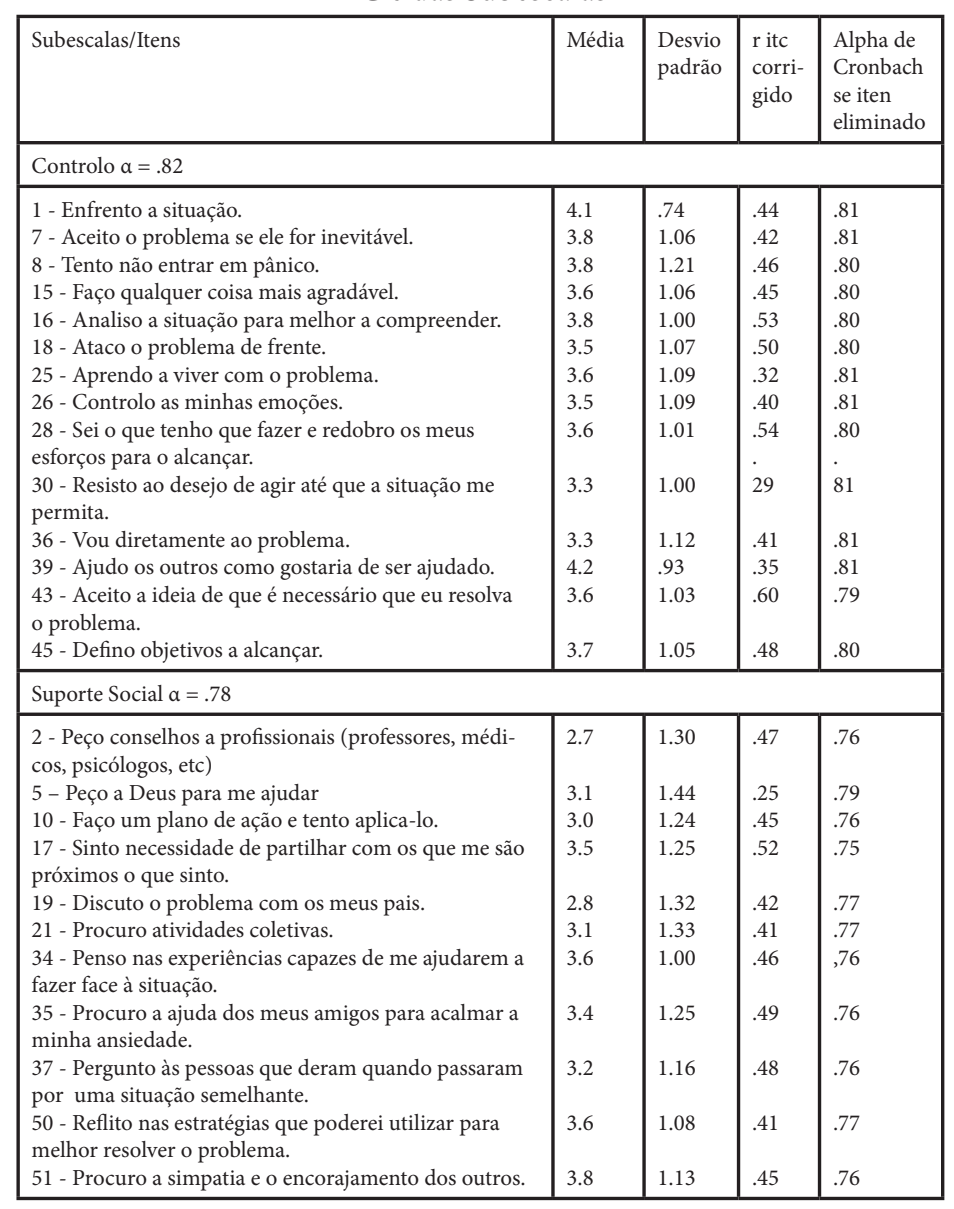

\begin{tabular}{|c|c|c|c|c|}
\hline \multicolumn{5}{|l|}{ Retraimento, Conversão e Aditividade $\alpha=.75$} \\
\hline 6 - Evito encontrar-me com pessoas. & 2.4 & 1.29 & .30 &, 75 \\
\hline 9 - Mudo a minha forma de viver. & 2.2 & 1.27 & .40 &, 74 \\
\hline $\begin{array}{l}11 \text { - Face a uma situação difícil, tenho difículdade em } \\
\text { encontrar palavras para dizer o que sinto. }\end{array}$ & 3.3 & 1.13 & .34 & .74 \\
\hline 14 - Sinto-me agressivo para com outras pessoas. & 2.5 & 1.23 & .25 &, 75 \\
\hline $\begin{array}{l}20 \text { - Esqueço os meus problemas tomando medica- } \\
\text { mentos. }\end{array}$ & 1.4 & .92 & .38 & .74 \\
\hline 22 - Refugio-me no imaginário ou no sonho. & 2.6 & 1.44 & .46 & .73 \\
\hline $\begin{array}{l}23 \text { - Procuro encontrar uma filosofia de vida (sentido, } \\
\text { valor) para fazer face à situação. }\end{array}$ & 3.0 & 1.21 & .32 & .74 \\
\hline 24 - Acontece-me não fazer o que tinha decidido. & 2.9 & 1.07 & .41 & .74 \\
\hline 27 - Mudo o meu comportamento. & 2.8 & 1.28 & .38 & .74 \\
\hline 32 - Sinto-me culpado. & 2.9 & 1.10 & .38 &, 74 \\
\hline $\begin{array}{l}38 \text { - Tomo drogas ou fumo para acalmar a minha } \\
\text { angústia. }\end{array}$ & 1.4 & .96 & .27 &, 75 \\
\hline 42 - Afasto-me dos outros. & 2.1 & 1.20 & .49 &, 73 \\
\hline 44 - Modifico as minhas ações em função do problema. & 3.0 & 1.16 & .32 & .74 \\
\hline 48 - Sinto-me invadido pelas minhas emoções. & 3.1 & 1.18 & .44 & .73 \\
\hline \multicolumn{5}{|l|}{ Distração Social $\alpha=.70$} \\
\hline $\begin{array}{l}3 \text { - Trabalho em conjunto com outras pessoas para me } \\
\text { esquecer. }\end{array}$ & 3.1 & 1.18 & .33 & .69 \\
\hline 4 - Tento não pensar no problema. & 3.6 & 1.20 & .41 & .67 \\
\hline 13 - Recuso-me a admitir o problema. & 2.4 & 1.21 & .28 & .71 \\
\hline $\begin{array}{l}33 \text { - Vou ao cinema, internet, vejo televisão ou faço } \\
\text { jogos para pensar menos nisso. }\end{array}$ & 3.7 & 1.17 & .52 & .63 \\
\hline 40 - Procuro a todo o custo pensar noutra coisa. & 3.6 & 1.11 & .56 & .62 \\
\hline 49 - Centro-me noutras atividades para me distrair. & 3.6 & 1.12 & .51 & .63 \\
\hline \multicolumn{5}{|l|}{ Recusa $\alpha=.69$} \\
\hline 12 - Não penso nos problemas quando eles se colocam. & 2.6 & 1.18 & .34 & .67 \\
\hline $\begin{array}{l}29 \text { - Chego a não sentir nada quando chegam as } \\
\text { dificuldades. }\end{array}$ & 2.8 & 1.07 & .38 & .66 \\
\hline 31 - Reajo como se o problema não existisse. & 2.6 & 1.27 & .43 & .65 \\
\hline 41 - Brinco com a situação em que me encontro. & 2.4 & 1.33 & .34 & .68 \\
\hline $\begin{array}{l}46 \text { - As minhas emoções desaparecem tão rapidamente } \\
\text { como aparecem. }\end{array}$ & 2.7 & 1.27 & .50 & .62 \\
\hline $\begin{array}{l}47 \text { - Digo a mim próprio que este problema não tem } \\
\text { importância. }\end{array}$ & 2.8 & 1.24 & .54 & .61 \\
\hline \multicolumn{4}{|l|}{ Alpha Global } & .85 \\
\hline
\end{tabular}

No nosso estudo a ETC apresentou um valor de Alfa de Cronbach global de .85 , superando o do estudo original, cujo Alfa foi de .78.(Tap, Costa e Alves, 2005). Na Tabela 3 comparamos os resultados nos domínios das subescalas da ETC entre o estudo desenvolvido em Portugal com uma população de jovens adultos e o atual (Tap, Costa e Alves, 2005), número de itens e valores de Alpha de Cronbach. Os resultados da análise de consistência interna da ETC indicam que os valores para cada uma das cinco subescalas encontrados na análise fatorial apresentam índices de consistência interna bons, com valores Alpha de Cronbach a variar entre .69 e .85 .

Tabela 3 - Comparação dos Domínios das Subescalas da ETC, Número de Itens e Valores de Alpha de Cronbach nos Dois Estudos

\begin{tabular}{|l|c|c|c|c|}
\hline \multirow{2}{*}{ Subescalas } & \multicolumn{2}{|c|}{ Estudo original } & \multicolumn{2}{c|}{ Estudo atual } \\
\cline { 2 - 5 } & $\begin{array}{c}\text { Número } \\
\text { de itens }\end{array}$ & $\begin{array}{c}\text { Alpha de } \\
\text { Cronbach }\end{array}$ & $\begin{array}{c}\text { Número } \\
\text { de itens }\end{array}$ & $\begin{array}{c}\text { Alpha de } \\
\text { Cronbach }\end{array}$ \\
\hline Controlo & 13 & .82 & 14 & .82 \\
\hline Suporte Social & 7 & .70 & 11 & .78 \\
\hline $\begin{array}{l}\text { Retraimento, Conversão e } \\
\text { Aditividade }\end{array}$ & 13 & .76 & 14 & .75 \\
\hline Recusa & 8 & .66 & 6 & .69 \\
\hline Distração Social & 10 & .72 & 6 & .70 \\
\hline
\end{tabular}


Para verificarmos a influência da variável género no Coping Global dos adolescentes, procedemos à comparação das respetivas médias nas subescalas da ETC ( $t$ test para amostras independentes). Da análise realizada, verificamos que o Coping Global é em média ligeiramente mais elevado nos rapazes $(M=159.4)$. As estratégias de controlo, suporte social e distração social são utilizadas de forma idêntica por rapazes e raparigas. Relativamente às estratégias de RCA são tendencialmente mais utilizadas pelas raparigas $(\mathrm{t}=-1.848 ; \mathrm{p}=0.066)$ e a recusa pelos rapazes $(t=4.571 ; \mathrm{p}=0.000)$, sendo estatisticamente significativa (Tabela 4 ).

Tabela 4 - Resultados nas Subescalas da ETC em Função do Género

\begin{tabular}{|c|c|c|c|c|c|c|}
\hline Subescalas & Sexo & $\mathrm{N}$ & Média & DP & $\mathrm{t}$ & $\mathrm{p}$ \\
\hline \multirow{2}{*}{ Coping Global } & Masculino & 124 & 159.4 & 23.81 & \multirow{2}{*}{.570} & \multirow{2}{*}{.569} \\
\hline & Feminino & 167 & 158.0 & 17.19 & & \\
\hline \multirow{2}{*}{ Controlo } & Masculino & 124 & 51.6 & 8.40 & \multirow{2}{*}{.179} & \multirow{2}{*}{.858} \\
\hline & Feminino & 167 & 51.4 & 7.47 & & \\
\hline \multirow{2}{*}{ Suporte Social } & Masculino & 124 & 36.0 & 8.35 & \multirow{2}{*}{.342} & \multirow{2}{*}{.733} \\
\hline & Feminino & 167 & 35.7 & 6.96 & & \\
\hline \multirow{2}{*}{$\begin{array}{l}\text { Retraimento, Conversão } \\
\text { e Aditividade }\end{array}$} & Masculino & 124 & 34.5 & 8.96 & \multirow{2}{*}{-1.848} & \multirow{2}{*}{.066} \\
\hline & Feminino & 167 & 36.3 & 7.27 & & \\
\hline \multirow{2}{*}{ Recusa } & Masculino & 124 & 17.4 & 4.62 & \multirow{2}{*}{4.571} & \multirow{2}{*}{.000} \\
\hline & Feminino & 167 & 14.9 & 4.33 & & \\
\hline \multirow{2}{*}{ Distração Social } & Masculino & 124 & 20.0 & 4.28 & \multirow{2}{*}{.457} & \multirow{2}{*}{.648} \\
\hline & Feminino & 167 & 19.7 & 4.52 & & \\
\hline
\end{tabular}

Para compararmos as médias nas subescalas da ETC com a idade, constituímos dois grupos de adolescentes segundo o grupo etário (GI - participantes dos 12 aos 14 anos e GII - participantes dos 15 aos 18 anos). Quando relacionadas as subescalas com a idade não se observaram diferenças estatisticamente significativas nas estratégias de Coping entre alunos mais novos e mais velhos. No entanto, constatamos que o grupo de alunos mais novos obteve no Coping Global uma média ligeiramente superior $(M=158,9)$, conseguida essencialmente à custa de maior utilização das estratégias de controlo e recusa (Tabela 5).

Tabela 5 - Resultados nas Subescalas da ETC em Função da Idade

\begin{tabular}{|c|c|c|c|c|c|c|}
\hline Subescalas & Grupo Etário & $\mathrm{N}$ & Média & $\mathrm{DP}$ & $\mathrm{t}$ & $\mathrm{p}$ \\
\hline \multirow{2}{*}{ Coping Global } & GI & 241 & 158.9 & 20.62 & \multirow{2}{*}{.497} & \multirow{2}{*}{.619} \\
\hline & GII & 50 & 157.3 & 18.47 & & \\
\hline \multirow{2}{*}{ Controlo } & G I & 241 & 51.7 & 8.22 & \multirow{2}{*}{.949} & \multirow{2}{*}{.344} \\
\hline & G II & 50 & 50.5 & 5.86 & & \\
\hline \multirow{2}{*}{ Suporte Social } & G I & 241 & 35.8 & 7.69 & \multirow{2}{*}{.289} & \multirow{2}{*}{.851} \\
\hline & G II & 50 & 35.6 & 7.02 & & \\
\hline \multirow{2}{*}{$\begin{array}{l}\text { Retraimento, Conversão } \\
\text { e Aditividade }\end{array}$} & G I & 241 & 35.5 & 8.17 & \multirow{2}{*}{-.302} & \multirow{2}{*}{.763} \\
\hline & G II & 50 & 35.8 & 7.61 & & \\
\hline \multirow{2}{*}{ Recusa } & G I & 241 & 16.1 & 4.58 & \multirow{2}{*}{.926} & \multirow{2}{*}{.355} \\
\hline & G II & 50 & 15.4 & 4.74 & & \\
\hline \multirow{2}{*}{ Distração Social } & G I & 241 & 19.8 & 4.50 & \multirow{2}{*}{-.143} & \multirow{2}{*}{886} \\
\hline & G II & 50 & 19.9 & 3.96 & & \\
\hline
\end{tabular}

\section{DISCUSSÃO}

Comparativamente ao estudo original, (Tap, et al., 2005), verificamos a manutenção das subescalas e todos os itens, embora com uma distribuição ligeiramente diferente, provavelmente pelo facto dos participantes serem mais jovens; o valor global de Alpha de Cronbach encontrado (.85) é superior ao estudo da versão original (.78); as estratégias de Controlo obtiveram um valor igual (.82); o Suporte Social (.78/.70) e a Recusa (.69/.66) apresentaram valores superiores; o RCA (.75/.76) e a Distração Social (.70/.72) valores inferiores.

As comparações de médias entre as estratégias de Coping e as variáveis género e idade também revelaram diferenças importantes em relação ao estudo original (Tap, et al., 2005).

Relativamente aos resultados do estudo original com jovens adultos, os adolescentes deste estudo utilizam mais estratégias de Coping Global, quer sejam do sexo masculino (159.4/146.7), quer do sexo feminino (158.0/150.7). Esta constatação poderá ter relação com a fase de desenvolvimento em que se encontram os adolescentes. Ou seja, um processo caraterizado por mudanças importantes a nível físico, social, psicológico e cognitivo ao mesmo tempo que procuram a sua autonomia e identidade, escolhem o seu caminho profissional e o seu projeto de vida (Cunha e Marques, 2009; Ferreira e Nelas, 2006), podendo as tarefas para o desenvolvimento acarretar níveis de stress significativos (Neuenschwander, 2002). Contudo, apesar dos adolescentes deste estudo utilizarem mais estratégias de Coping que os jovens adultos (Tap, et al., 2005), o estudo da variável idade não apresenta diferenças estatisticamente significativas em relação a nenhuma das subescalas da ETC, contrariamente ao estudo original (Tap, et al., 2005) e a outro estudo desenvolvido em Portugal com adolescentes que, embora utilizando instrumentos diferentes, concluiu que as estratégias de Coping aumentam com a idade (Borges, Manso, Tomé e Matos, 2008).

$\mathrm{Na}$ análise da variável sexo, apenas a estratégia Recusa apresenta significância estatística favorável aos rapazes, contrariamente ao estudo original (Tap, et al., 2005) em que o Coping Global e as restantes estratégias apresentam significância estatística exceto a estratégia de Recusa que apenas apresenta tendência de maior utilização pelos participantes do sexo masculino (Tap, et al., 2005). Relativamente às raparigas, embora sem significância estatística, os resultados deste estudo mostram que estas utilizam mais estratégias de RCA para enfrentar as adversidades, o que está em consonância com o estudo original (Tap, et al., 2005). 
Na tentativa de justificar o porquê das diferenças entre rapazes e raparigas, encontramos um estudo que aponta diferenças na educação de rapazes e raparigas e alerta para a possibilidade de adoção de comportamentos de risco na tentativa de gestão das adversidades (Câmara e Carloto, 2007).

Ainda, segundo os mesmos autores, os rapazes utilizam mais estratégias de recusa, e as raparigas estratégias mais relacionadas com as emoções. Também, os traços de personalidade dos adolescentes relacionam-se significativamente com as estratégias de coping (Diniz et al., 2010).

Outro estudo sobre coping, desenvolvido com adolescentes diabéticos, mostra que as estratégias de recusa estão relacionadas com menor competência e menor controlo metabólico (Jaser \& White, 2010).

\section{CONCLUSÃO}

A Escala Toulousiana de Coping mantém as qualidades métricas do estudo original realizado por Tap, Costa e Alves, em 2005, considerando-se por isso um instrumento fiável. O estudo psicométrico da ETC junto de adolescentes dos 12 aos 18 anos, à semelhança do estudo de adaptação e validação para a cultura portuguesa, permitiu encontrar 5 dimensões ou subescalas: controlo; suporte social; RCA; distração social e recusa. Neste estudo o valor do coeficiente Alpha de Cronbach para o instrumento na sua globalidade é de .85 , superando o do estudo original (.78).
Ao compararmos os valores médios obtidos pelos adolescentes (participantes deste estudo) e os jovens (participantes no estudo de adaptação e validação da escala para a cultura portuguesa), encontramos algumas diferenças que poderão ser justificadas pelo fator idade.

\section{IMPLICAÇÕES PARA A PRÁTICA CLÍNICA}

Num mundo da saúde altamente tecnológico onde a informação produzida sustenta grande parte das decisões, importa produzir conhecimento que favoreça uma prestação de cuidados de saúde mais personalizados. A ETC é um instrumento fiável e pode ser aplicado a adolescentes a fim de produzir informação das estratégias de coping mais utilizadas face às dificuldades com que se vão confrontando. Este conhecimento deverá sustentar as intervenções de saúde, tornando o cuidado mais incisivo e adequado a cada adolescente com o intuito de promover o seu bem-estar físico, psicológico e qualidade de vida num desenvolvimento que se pretende saudável. Em ambiente escolar os adolescentes desenvolvem o seu processo de socialização. A aplicação da ETC permite perceber as estratégias de coping mais usuais, podendo servir de base a projetos de intervenção que ajudem o adolescente a descobrir competências pessoais que permitam lidar melhor com as adversidades.

Fica disponível um instrumento de medida confiável para a investigação em adolescentes podendo ser relacionado com múltiplas situações das quais destacamos a diabetes, a obesidade, as dependências aditivas e os comportamentos desviantes.

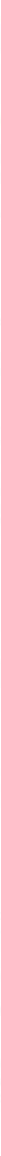




\section{REFERÊNCIAS BIBLIOGRÁFICAS}

Almeida, L., e Freire, T. (2008). Metodologia da investigação em psicologia e educação ( $5^{\mathrm{a}}$ ed.). Braga: Psiquilíbrios.

Alves, M. N. (2003). Les émigrés de retour au Portugal: Attitudes, stress et stratégies de coping. Tese de Doutoramento, Université de Toulouse-Le-Mirail, França.

Antoniazzi, A. S., Dell'Aglio, D. D., e Bandeira, D. R. (1998). O Conceito de coping: Uma revisão teórica. Estudos de Psicologia, 3(2), 273-294.

Borges, A.I., Manso, D.S., Tomé, G., Matos, M.G. (2008) Ansiedade e coping em crianças e adolescentes: diferenças relacionadas com a idade e género. Análise Psicológica, 4(26), 551-561. Acedido em http://www.scielo. mec.pt/pdf/aps/v26n4/v26n4a02.pdf

Câmara, S., e Carloto, M. S. (2007). Coping e género em adolescentes. Psicologia em Estudo, 12(1), 87-93.

Cunha, I., e Marques, E. (2009). A Construção do eu adolescente na relação com o(s) outro(s): O igual, o diferente e o complementar através do Rorschach. Análise Psicológica, 3(28), 247-257. Acedido em http://www. scielo.mec.pt/pdf/aps/v27n3/v27n3a03.pdf

Diniz, S. S., e Zanini, D. S. (2010). Relação entre fatores de personalidade e estratégias de coping em adolescentes. Psicologia - Universidade de Sâo Francisco, 15(1), 71-80.

Esparbès, S., Sordes-Ades, F., \& Tap, P. (1993). Presentación de l'echelle de coping. Actes de las Journées du Laboratoire Personnalisation et Changements Sociaux: Saint Criq: Université de Toulouse-Le Mirail, 89-107.

Faro, A., e Pereira, M. (2013). Estresse: Revisão narrativa da evolução conceitual, perspetivas teóricas e metodológicas. Psicologia, Saúde e Doenças, 14(1), 78100. Acedido em http://www.scielo.mec.pt/pdf/psd/ v14n1/v14n1a06.pdf

Ferreira, M. F., e Nelas, P. B. (2006). Adolescências... Adolescentes. Educação, Ciência e Tecnologia. Acedido em http://repositorio.ipv.pt/handle/10400.19/409
Jaser, S. S., \& White, L. E. (2010). Coping and resilience in adolescents with type 1 diabetes. Child: care, health and development, 37(3), 335-342. Acedido em http:// www.ncbi.nlm.nih.gov/pmc/articles/PMC3134245/

Lazarus, R. S., \& Folkman, S. (1984). Stress, appraisal and coping. New York: Springer.

Lazarus, R. S. (1992). Coping with the stress of illness. Who Regional Publications European Series, 44, 11-31.

Neuenschwander, M. (2002). Desenvolvimento e identidade na adolescência Coimbra: Almedina

Nunes, M. O. (2001). Drogues, valeurs et communautés (valeurs, coping, autonomie et sociabilité). Thèse de doctorat NR, Université de Toulouse-Le-Mirail.

Pais-Ribeiro, J. L. (2004). Questões acerca do coping: A propósito do estudo de adaptação do Brief COPE. Colecção Psicologia Saúde e Doenças, 5(1), 3-15.

Pais-Ribeiro, J. L. (2010). Metodologias de investigação em psicologia da saúde ( $3^{a}$ ed.). Oliveira de Azeméis, Portugal: Livpsic.

Pestana, M. H., e Gageiro, J. N. (2008). Análise de dados para ciências sociais: a complementaridade do SPSS. Lisboa: Edições Sílabo.

Tap, P., Costa, E. S., e Alves, M. N. (2005). Escala Toulousiana de Coping (ETC): Estudo de adaptação à população portuguesa. Psicologia Saúde e Doenças, 6(1), 47-56. Acedido em http://www.scielo.mec.pt/pdf/psd/ v6n1/v6n1a03.pdf

\section{AGRADECIMENTOS}

Aos Professores: Filomena Vieira e Graça Vilela pelas autorizações para efetuar a colheita de dados; Carla Leite, e Luís Sá pela colaboração na organização da colheita de dados.

À aluna da Licenciatura em Enfermagem, Bárbara Lucas, e à Professora Carla Leite pela aplicação dos questionários. 